\title{
Arteriovenous malformation of the trigeminal nerve root presented with venous congestive edema of the medulla oblongata and upper cervical cord: illustrative case
}

\author{
Arata Nagai, MD, ${ }^{1}$ Hidenori Endo, MD, PhD, ${ }^{1,2}$ Kenichi Sato, MD, PhD, ${ }^{3}$ Tomohiro Kawaguchi, MD, PhD, ${ }^{1}$ Hiroki Uchida, MD, PhD, ${ }^{1}$ \\ Shunsuke Omodaka, MD, PhD, ${ }^{4}$ Yasushi Matsumoto, MD, PhD, ${ }^{4}$ and Teiji Tominaga, MD, $\mathrm{PhD}{ }^{5}$

\begin{abstract}
${ }^{1}$ Department of Neurosurgery, Kohnan Hospital, Sendai, Japan; ${ }^{2}$ Division of Advanced Cerebrovascular Surgery, Tohoku University Graduate School of Medicine, Sendai, Miyagi, Japan; ${ }^{3}$ Department of Neurosurgery, Tohoku Medical and Pharmaceutical University Hospital, Sendai, Japan; ${ }^{4}$ Department of Neuroendovascular Therapy, Kohnan Hospital, Sendai, Japan; and ${ }^{5}$ Department of Neurosurgery, Tohoku University Graduate School of Medicine, Sendai, Japan
\end{abstract}

\begin{abstract}
BACKGROUND Arteriovenous malformation (AVM) of the trigeminal nerve root (TNR) is a rare subtype of the lateral pontine AVM. Most of them are diagnosed when they bleed or exert trigeminal neuralgia. Venous congestive edema is a rare phenomenon caused by TNR AVMs.

OBSERVATIONS An 82-year-old man was admitted with progressive limb weakness and dysphasia. Magnetic resonance imaging (MRI) revealed extensive edema of the medulla oblongata and the upper cervical cord with signal flow void at the C3 anterior spinal cord. Vertebral angiography revealed a small nidus fed mainly by the pontine perforating arteries (PPAs). The anterior pontomesencephalic vein (AMPV) was dilated, functioning as the main drainage route. This suggests that venous hypertension triggered the brainstem and upper cervical cord edema. MRI with gadolinium enhancement showed that the nidus was located around the right TNR. Because the nidus sat extrinsically on the pial surface of the right TNR's base, microsurgical obliteration with minimum parenchymal injury was achieved. Postoperative MRI showed disappearance of the brainstem and cervical cord edema with improved clinical symptoms.
\end{abstract}

LESSONS TNR AVM is rarely associated with brainstem and upper cervical cord edema caused by venous hypertension of the congestive drainage system.

https://thejns.org/doi/abs/10.3171/CASE21402

KEYWORDS arteriovenous malformation; brainstem; trigeminal nerve root; venous congestion; brainstem edema

Brainstem arteriovenous malformations (AVMs) are challenging to treat because they are located in one of the inviolable areas of the brain, including the cranial nerves, their nuclei, and critical fiber tracts, ${ }^{1}$ and have a more aggressive clinical course than those in other locations. Their clinical manifestation includes hemorrhage, mass effect, or neurovascular compression syndrome. ${ }^{2-8}$ These lesions can be treated with a less invasive strategy, such as Gamma Knife or endovascular embolization.

Here, we report a case of lateral pontine AVM located at the base of the right trigeminal nerve root (TNR) with progressing edema of the medulla oblongata and upper cervical cord, successfully treated with open surgery.

\section{Illustrative Case}

An 82-year-old man presented with a 2-month history of progressive limb weakness, dysarthria, dysphagia, and urinary incontinence. T2-weighted magnetic resonance imaging (MRI) revealed extensive swelling and hyperintensity of the medulla oblongata and upper cervical spinal cord (Fig. 1A) and signal flow void at the C3 anterior spinal cord. This suggests the existence of vascular

ABBREVIATIONS AICA = anterior inferior cerebellar artery; AMPV = anterior pontomesencephalic vein; $\mathrm{AVM}=$ arteriovenous malformation; $\mathrm{CPAC}=$ cerebellopontine angle cistern; DAVS = dural arteriovenous shunt; ICG = indocyanine green; MRI = magnetic resonance imaging; PPA = pontine perforating artery; SPS = superior petrosal sinus; TNR $=$ trigeminal nerve root; TPV $=$ transverse pontine vein

INCLUDE WHEN CITING Published August 30, 2021; DOI: 10.3171/CASE21402

SUBMITTED July 13, 2021. ACCEPTED July 26, 2021.

(C) 2021 The authors, CC BY-NC-ND 4.0 (http://creativecommons.org/licenses/by-nc-nd/4.0/). 
abnormalities of the upper cervical spine. Bilateral vertebral artery angiography revealed no upper cervical spine abnormal findings. Left vertebral angiograms showed a small intracranial AVM fed by a dilated pontine perforating artery (PPA) arising from the upper basilar artery and the anterior inferior cerebellar artery (AICA) (Fig. 1B and $\mathrm{C}$ ). The draining route was composed of a transverse pontine vein (TPV) draining into the anterior pontomesencephalic vein (APMV) (Fig. 1C and D). The petrosal vein and the superior petrosal sinus (SPS) were not apparent. Spoiled gradient recalled acquisition in the steady state with gadolinium enhancement revealed a nidus located around the right lateral pons, especially around the right TNR (Fig. 1E).

Open surgery was performed using the retrosigmoid approach. During surgery, a small nidus was found on the pial surface at the right TNR's posterior base (Fig. 2A and B). Using a neuroendoscope, we identified the deep feeder running up behind the TNR (Fig. 2C). The vascular caliber changed at the origin of the red draining vein, considered the petrosal vein. The draining vein turned back at the connection to the SPS, which drained into the dilated APMV via the TPV. SPS was not transparent on the petrous dural surface. We simply coagulated the nidus to occlude the shunt with minimum parenchymal dissection (Fig. 2D). Indocyanine green (ICG) video angiography revealed shunt flow disappearance (not shown).

The patient's neurological symptoms gradually improved after surgery, except for bladder and bowel dysfunction. A postoperative
MRI study revealed resolution of the T2-hyperintense signal, swelling of the spinal cord, and abnormal dilated spinal vein disappearance (Fig. 3A). Follow-up angiography performed 1 month after surgery confirmed shunt disappearance (Fig. 3B). The patient was transferred to a rehabilitation service 2 months after surgery.

\section{Discussion}

The present case had extensive medulla oblongata and upper cervical cord edemas with flow void signal of the anterior spinal veins at the C3 spinal level. These MRI findings suggested the existence of vascular abnormalities of the upper cervical spine. However, we found a small lateral pontine AVM located at the pial layer of the base of the TNR. This is a rare clinical onset pattern of TNR AVM.

\section{Observations}

The present case had a small nidus primarily located at the pial layer of the TNR, which was fed by the PPA and the AICA and originally provided arterial supply for the TNR. ${ }^{9}$ The present case's angioarchitecture was similar to that of the AVM in the cerebellopontine angle cistern (CPAC). ${ }^{10}$ Among the CPAC AVMs, a total of 34 cases were associated with the TNR (Table 1). ${ }^{2-8,10-18}$ TNR AVM occurs predominantly among early-middle- to late-middleaged males, often presenting with hemorrhage or trigeminal neuralgia. This is the first case that presented as TNR AVM with brainstem edema. The main draining routes are usually composed of a
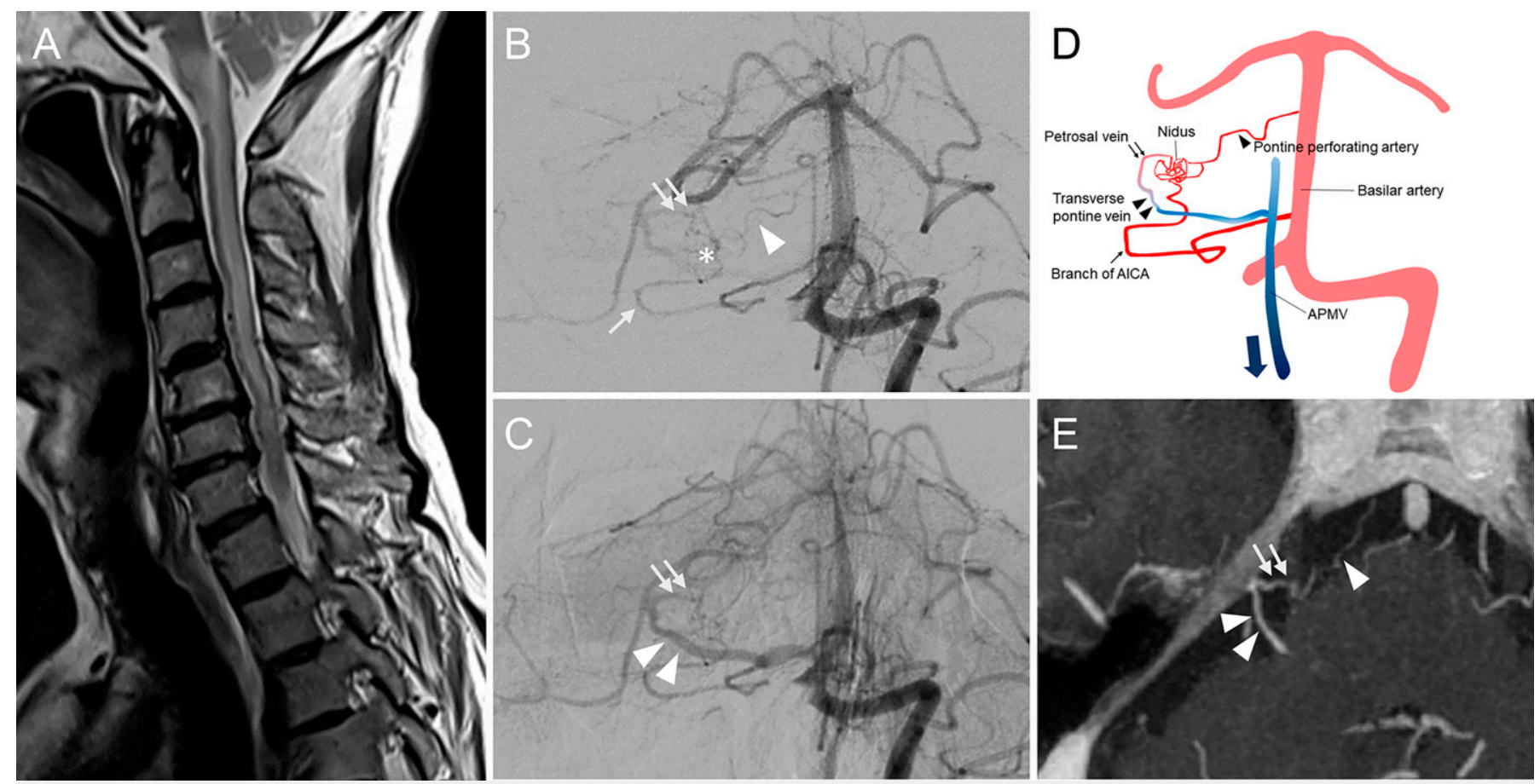

FIG. 1. A: Preoperative sagittal T2-weighted MRI scan revealing swelling and abnormal intensity of the medulla oblongata and upper cervical spinal cord. B: The arterial phase of preoperative angiography showing the small AVM (asterisk). The feeding system was composed of the AICA (arrow) and the PPA (arrowhead). Double arrows show the venous drainage origin. C: The late arterial phase of preoperative angiography showing TPV (double arrowheads) as the main draining route into the AMPV. Double arrows show the venous drainage origin. D: Schematic illustration of the preoperative angiographic interpretation. E: Preoperative spoiled gradient recalled acquisition in the steady-state with gadolinium enhancement revealing the PPA (feeder; arrowhead), the petrosal vein (drainer; double arrows), and the TPV (drainer; double arrowheads) around the right TNR. Each arrow and arrowhead in A-E shows the same vascular structures. 

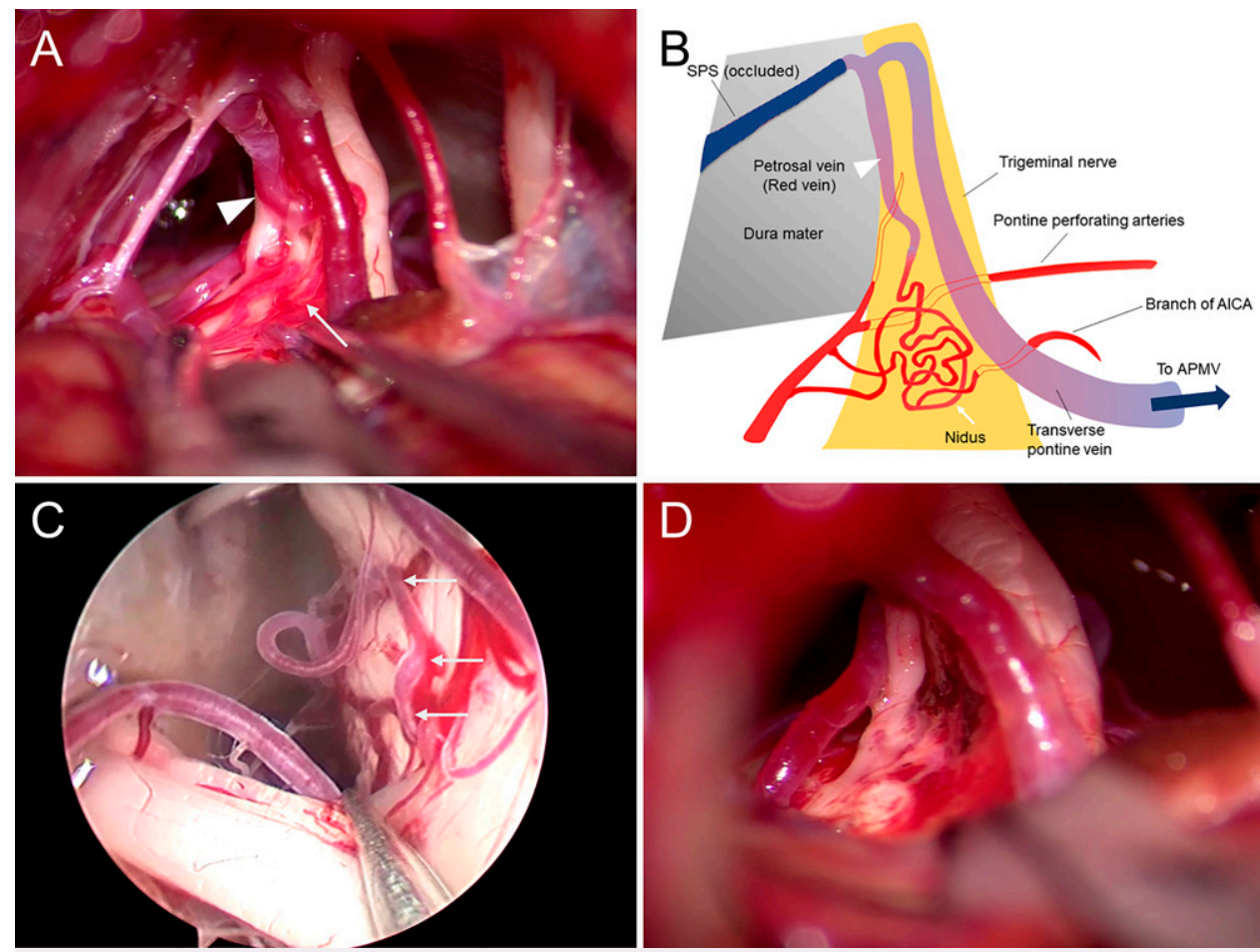

FIG. 2. A: Intraoperative photograph showing the small nidus (arrow) and the drainer (arrowhead). B: Operative field illustration. C: Neuroendoscopic view from infratrigeminal window showing the feeder running up from the deep (triple arrows). D: Final view of the operation showing the coagulated nidus and TNR preservation.
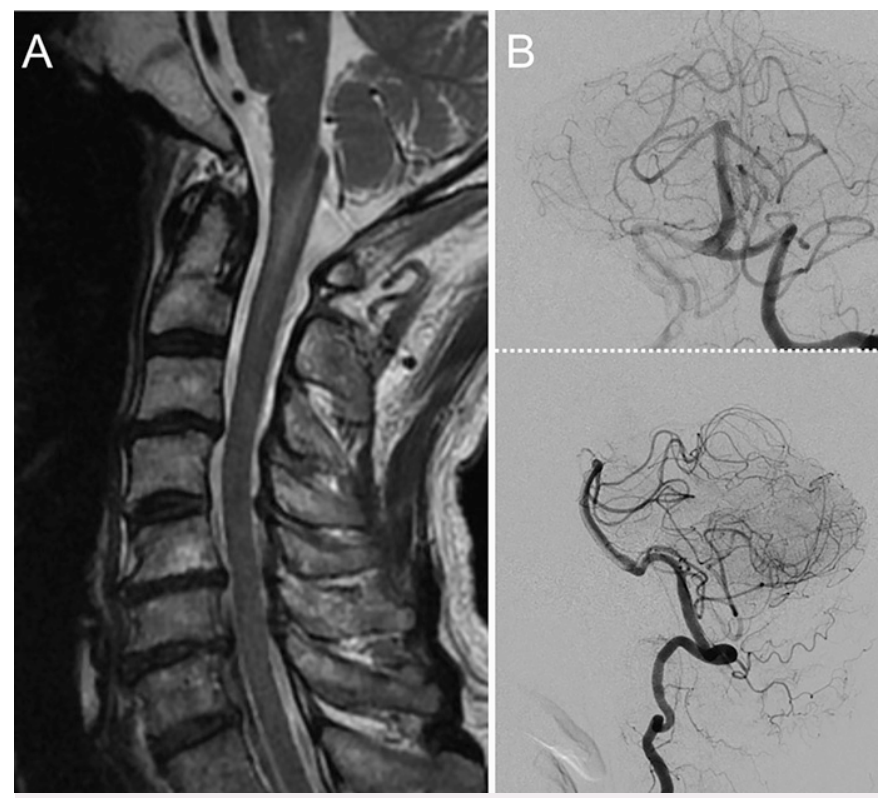

FIG. 3. A: Sagittal T2-weighted MRI scan 1 month after treatment showing marked resolution of the medulla oblongata and the upper cervical spinal cord swelling and edema. B: Follow-up angiography performed 1 month after treatment showing no residual shunt. 


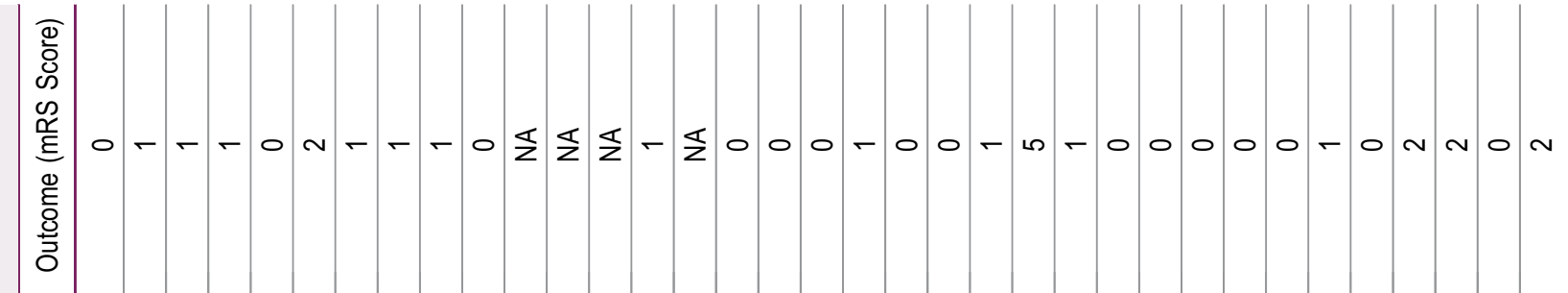

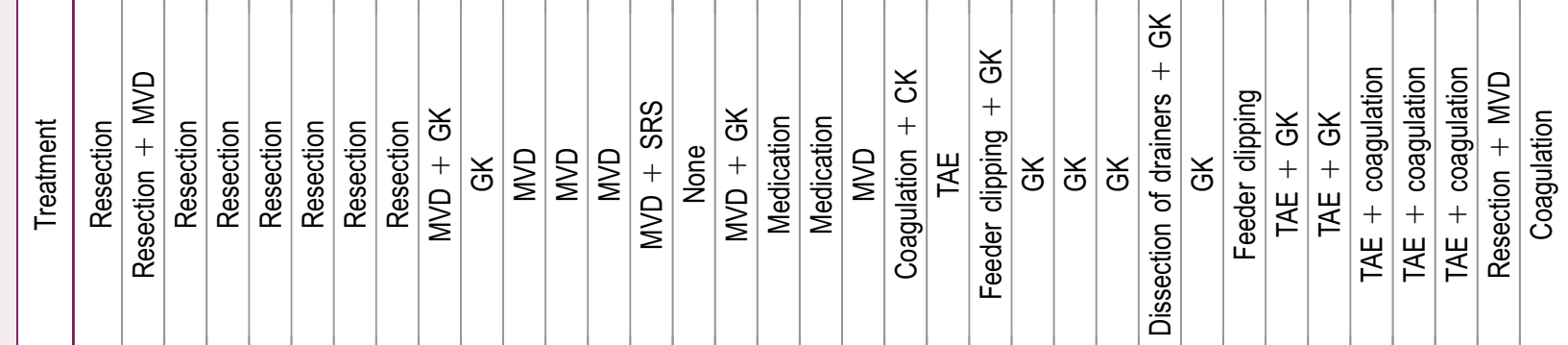

重焉

is

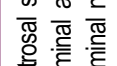

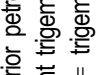

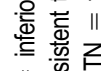

is

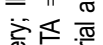

행

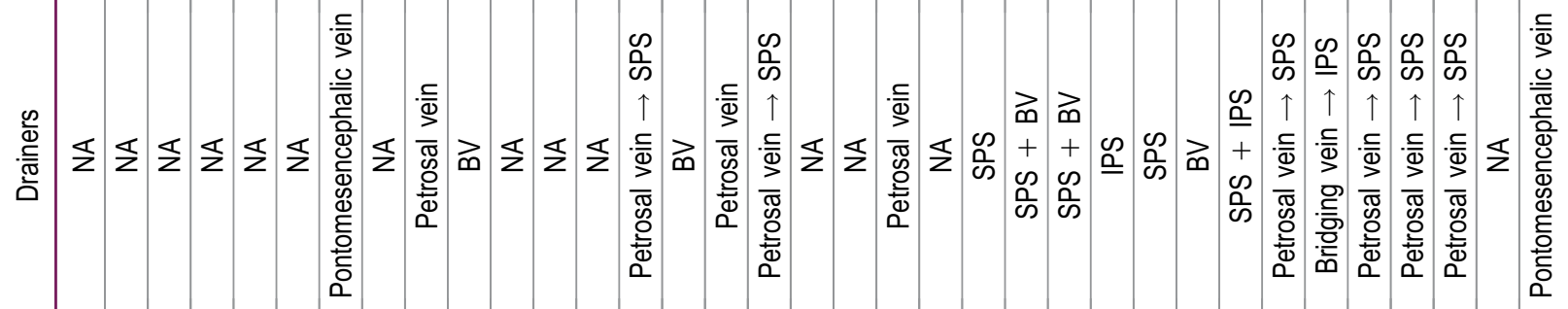

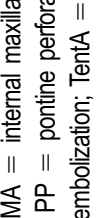

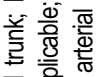

징

흥 흔

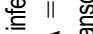
II $\frac{\pi}{5}$ 豆额崖

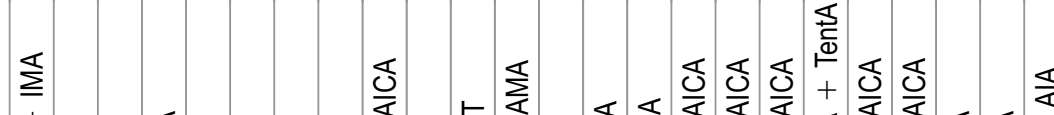

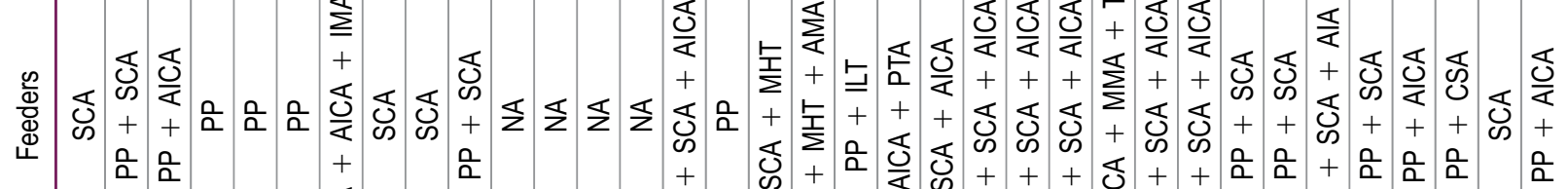
항 흘 용 즌 흔

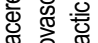

它

a

음

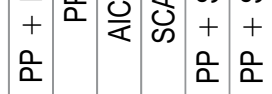

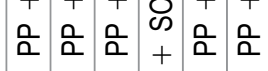

음

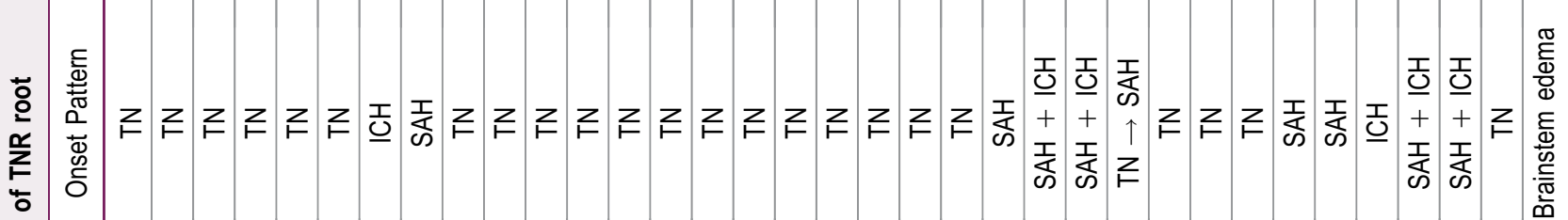

๑ 寸

$\cos 2 \operatorname{lon}$

$2 \sqrt{2}$

œ

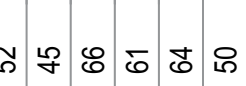

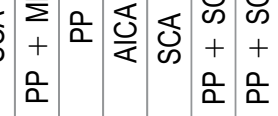

a

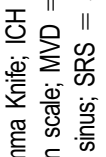

衰

爻数

을 을

离

今。

挤离

要

急离

京竞 $\frac{0}{\overline{0}}$

产

离

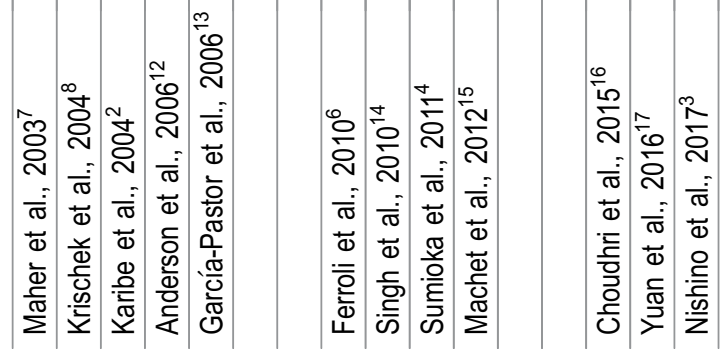

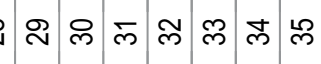

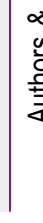

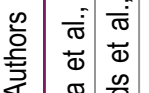

ర్స = ర

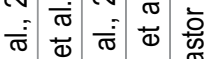

๑

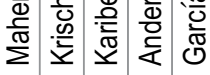

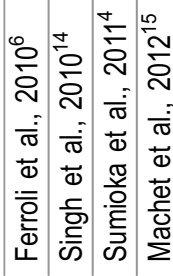

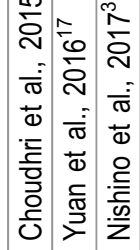

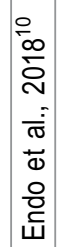

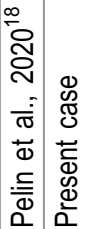


petrosal vein that drains into the SPS. However, in the present case, the draining route was composed of a TPV draining into the APMV. The SPS was invisible on both a preoperative angiogram and by intraoperative observation. It might have functioned as the main drainage route the thrombotic occlusion of which resulted in APMV induced as a new venous drainage route. The present single-case report cannot explain the exact mechanisms underlying the drainage route's thrombosis. The mechanical action of pressure and shearing stress to the endothelial surface was supposed to be produced by the shunt blood flow causing the subsequent vessel wall hyperplasia, thrombosis, and luminal narrowing. ${ }^{19,20}$ The effect of aging might be associated with drainage route obstruction because the present case is the oldest among the TNR AVM reported cases.

In the present case, increased pressure within the APMV affected the normal venous drainage of the medulla oblongata and upper cervical cord, causing venous congestion and widespread edema. Likewise, an intracranial dural arteriovenous shunt (DAVS) with spinal venous drainage often presents with progressive myelopathy caused by spinal venous system congestion. Arteriovenous shunt-induced blood flow increase leads to increased venous pressure, reducing the arteriovenous pressure gradient, causing decreased brainstem and spinal cord perfusion. ${ }^{21}$ The prompt diagnosis of these arteriovenous shunts is sometimes difficult because MRI findings are similar to those of myelitis, infarction, and intramedullary tumors. ${ }^{22}$ Haryu et al. ${ }^{23}$ indicated the flow void signals on sagittal T2-weighted MRI as an effective diagnostic marker of intracranial DAVS with spinal venous drainage. In the present case, the flow void signal on MRI was also the intracranial arteriovenous shunt diagnostic clue.

Lateral pontine AVMs sometimes grow extrinsically on the pial surface rather than intrinsically in the parenchyma. ${ }^{1,3,10,24}$ As demonstrated by Hoh et al.., ${ }^{25}$ shunt disconnection without resection of the intraparenchymal component was sufficient for this type of lateral pontine AVM. Surgical failure was seen in only $7.8 \%$ of cases. We applied this "occlusion in situ" technique to the present case's treatment. We coagulated the feeding arteries on the pial surface and minimized the parenchymal dissection extent, allowing neurological function preservation. The color change in the draining vein and the ICG video angiography findings proved shunt elimination. ${ }^{6}$

\section{Lessons}

TNR AVMs usually manifest hemorrhage or neurovascular compression syndrome..$^{2-8}$ Venous congestive edema of the medulla oblongata and the upper cervical cord is a rare onset pattern of TNR AVMs. TNR AVM could be a differential diagnosis of the brainstem or upper cervical cord edema.

\section{Acknowledgments}

We thank Enago for English-language proofreading.

\section{References}

1. Nozaki K, Hashimoto N, Kikuta K, Takagi Y, Kikuchi H. Surgical applications to arteriovenous malformations involving the brainstem. Neurosurgery. 2006;58(4 suppl 2):ONS-270-ONS-279.

2. Karibe $H$, Shirane R, Jokura $H$, Yoshimoto $T$. Intrinsic arteriovenous malformation of the trigeminal nerve in a patient with trigeminal neuralgia: case report. Neurosurgery. 2004;55(6):1433.
3. Nishino K, Hasegawa H, Morita K, et al. Clinical characteristics of arteriovenous malformations in the cerebellopontine angle cistern. $J$ Neurosurg. 2017;126(1):60-68.

4. Sumioka S, Kondo A, Tanabe H, Yasuda S. Intrinsic arteriovenous malformation embedded in the trigeminal nerve of a patient with trigeminal neuralgia. Neurol Med Chir (Tokyo). 2011;51(9):639-641.

5. Edwards RJ, Clarke Y, Renowden SA, Coakham HB. Trigeminal neuralgia caused by microarteriovenous malformations of the trigeminal nerve root entry zone: symptomatic relief following complete excision of the lesion with nerve root preservation. $J$ Neurosurg. 2002;97(4):874-880.

6. Ferroli P, Acerbi F, Broggi M, Broggi G. Arteriovenous micromalformation of the trigeminal root: intraoperative diagnosis with indocyanine green videoangiography: case report. Neurosurgery. 2010;67(3 Suppl Operative):E309-E310.

7. Maher CO, Atkinson JL, Lane Jl. Arteriovenous malformation in the trigeminal nerve. Case report. J Neurosurg. 2003;98(4):908-912.

8. Krischek B, Yamaguchi S, Sure U, Benes L, Bien S, Bertalanffy H. Arteriovenous malformation surrounding the trigeminal nerve-case report. Neurol Med Chir (Tokyo). 2004;44(2):68-71.

9. Marinković S, Gibo H, Nikodijević I. Trigeminocerebellar arteryanatomy and possible clinical significance. Neurol Med Chir (Tokyo). 1996;36(4):215-219.

10. Endo H, Osawa SI, Matsumoto $\mathrm{Y}$, et al. Embolization of ruptured arteriovenous malformations in the cerebellopontine angle cistern. Neurosurg Rev. 2018;41(1):173-182.

11. Nomura T, Ikezaki K, Matsushima T, Fukui M. Trigeminal neuralgia: differentiation between intracranial mass lesions and ordinary vascular compression as causative lesions. Neurosurg Rev. 1994;17(1):51-57.

12. Anderson WS, Wang PP, Rigamonti D. Case of microarteriovenous malformation-induced trigeminal neuralgia treated with radiosurgery. $J$ Headache Pain. 2006;7(4):217-221.

13. García-Pastor C, López-González F, Revuelta R, Nathal E. Trigeminal neuralgia secondary to arteriovenous malformations of the posterior fossa. Surg Neurol. 2006;66(2):207-211.

14. Singh N, Bharatha A, O'Kelly C, et al. Intrinsic arteriovenous malformation of the trigeminal nerve. Can J Neurol Sci. 2010;37(5):681-683.

15. Machet A, Aggour M, Estrade L, Chays A, Pierot L. Trigeminal neuralgia related to arteriovenous malformation of the posterior fossa: three case reports and a review of the literature. J Neuroradiol. 2012;39(1):64-69.

16. Choudhri O, Heit JJ, Feroze AH, Chang SD, Dodd RL, Steinberg GK. Persistent trigeminal artery supply to an intrinsic trigeminal nerve arteriovenous malformation: a rare cause of trigeminal neuralgia. J Clin Neurosci. 2015;22(2):409-412.

17. Yuan Y, Zhang Y, Luo QI, Yu J. Trigeminal neuralgia caused by brain arteriovenous malformations: A case report and literature review. Exp Ther Med. 2016;12(1):69-80.

18. Pelin K, Göktuğ U, Yiğit A, Emre Y, Sukru A. Trigeminal neuralgia caused by cerebellar arteriovenous malformations: a case report and review of the literature. Neurol Sci Neurosurg. 2020;1(2):107.

19. Song JK, Patel AB, Duckwiler GR, et al. Adult pial arteriovenous fistula and superior sagittal sinus stenosis: angiographic evidence for high-flow venopathy at an atypical location. Case report. $J$ Neurosurg. 2002;96(4):792-795.

20. Osada $\mathrm{Y}$, Endo H, Sato K, et al. Successful presurgical endovascular management of venous sinus thrombosis associated with highgrade cerebral arteriovenous malformation: A case report. Interv Neuroradiol. 2017;23(6):656-660.

21. Wiesmann M, Padovan CS, Pfister HW, Yousry TA. Intracranial dural arteriovenous fistula with spinal medullary venous drainage. Eur Radiol. 2000;10(10):1606-1609. 
22. Kim NH, Cho KT, Seo HS. Myelopathy due to intracranial dural arteriovenous fistula: a potential diagnostic pitfall. Case report. $J$ Neurosurg. 2011;114(3):830-833.

23. Haryu S, Endo T, Sato K, Inoue T, Takahashi A, Tominaga T. Cognard type $V$ intracranial dural arteriovenous shunt: case reports and literature review with special consideration of the pattern of spinal venous drainage. Neurosurgery. 2014;74(1):E135-E142.

24. Han SJ, Englot DJ, Kim H, Lawton MT. Brainstem arteriovenous malformations: anatomical subtypes, assessment of "occlusion in situ" technique, and microsurgical results. J Neurosurg. 2015;122(1):107-117.

25. Hoh BL, Putman CM, Budzik RF, Ogilvy CS. Surgical and endovascular flow disconnection of intracranial pial single-channel arteriovenous fistulae. Neurosurgery. 2001;49(6):1351-1364.

\section{Disclosures}

Dr. Matsumoto reports personal fees from GE healthcare, Stryker Japan, Medico's Hirata, Medtronic Japan, Century Medica, Takeda
Pharmaceutical Limited, Otuka Pharmaceutical Limited, Kaneka Medics, and Fuji Systems, all outside the submitted work. No other disclosures were reported.

\section{Author Contributions}

Conception and design: Endo, Nagai, Uchida, Omodaka. Acquisition of data: Endo, Nagai, Sato, Kawaguchi, Uchida, Matsumoto. Analysis and interpretation of data: Endo, Nagai, Uchida, Matsumoto. Drafting the article: Endo, Nagai. Critically revising the article: Endo, Nagai, Sato, Omodaka. Reviewed submitted version of manuscript: Endo, Nagai, Sato, Uchida, Omodaka, Tominaga. Approved the final version of the manuscript on behalf of all authors: Endo. Study supervision: Omodaka, Tominaga.

\section{Correspondence}

Hidenori Endo: Kohnan Hospital, Miyagi, Japan. h-endo@nsg.med. tohoku.ac.jp. 